\title{
Successful scaling-up of self-sustained pyrolysis of oil palm biomass under pool-type reactor
}

\begin{abstract}
An appropriate technology for waste utilisation, especially for a large amount of abundant pressed-shredded oil palm empty fruit bunch (OFEFB), is important for the oil palm industry. Self-sustained pyrolysis, whereby oil palm biomass was combusted by itself to provide the heat for pyrolysis without an electrical heater, is more preferable owing to its simplicity, ease of operation and low energy requirement. In this study, biochar production under selfsustained pyrolysis of oil palm biomass in the form of oil palm empty fruit bunch was tested in a 3-t large-scale pool-type reactor. During the pyrolysis process, the biomass was loaded layer by layer when the smoke appeared on the top, to minimise the entrance of oxygen. This method had significantly increased the yield of biochar. In our previous report, we have tested on a $30-\mathrm{kg}$ pilot-scale capacity under self-sustained pyrolysis and found that the higher heating value (HHV) obtained was $22.6-24.7 \mathrm{MJ} \mathrm{kg}-1$ with a $23.5 \%-25.0 \%$ yield. In this scaled-up study, a 3-t large-scale procedure produced HHV of $22.0-24.3 \mathrm{MJ} \mathrm{kg}-1$ with a $30 \%-34 \%$ yield based on a wet-weight basis. The maximum self-sustained pyrolysis temperature for the large-scale procedure can reach between $600{ }^{\circ} \mathrm{C}$ and $700{ }^{\circ} \mathrm{C}$. We concluded that large-scale biochar production under self-sustained pyrolysis was successfully conducted owing to the comparable biochar produced, compared with medium-scale and other studies with an electrical heating element, making it an appropriate technology for waste utilisation, particularly for the oil palm industry.
\end{abstract}

Keyword: Biochar; Charcoal; Oil palm biomass; Self-sustained pyrolysis 